\title{
Padé summability of the cubic oscillator
}

\author{
Vincenzo Grecchi ${ }^{1}$ \\ Marco Maioli ${ }^{2}$ \\ André Martinez ${ }^{3}$
}

\begin{abstract}
.
We prove the Padé (Stieltjes) summability of the perturbation series of the energy levels of the cubic anharmonic oscillator, $H_{1}(\beta)=p^{2}+x^{2}+i \sqrt{\beta} x^{3}$, as suggested by the numerical studies of Bender and Weniger. At the same time, we give a simple and independent proof of the positivity of the eigenvalues of the $\mathcal{P} \mathcal{T}$-symmetric operator $H_{1}(\beta)$ for real $\beta$ (Bessis-Zinn Justin conjecture). All the $n \in \mathrm{N}$ zeros of an eigenfunction, real at $\beta=0$, become complex with negative imaginary part, for complex, non-negative $\beta \neq 0$.
\end{abstract}

PACS numbers: 03.65.Sq, 02.30.Lt, 03.65.Ge

\footnotetext{
${ }^{1}$ Dipartimento di Matematica, Università di Bologna, I-40127-Bologna, Italy - E.mail: grecchi@dm.unibo.it

${ }^{2}$ Dipartimento di Matematica, Università di Modena, I-41100-Modena, Italy - E.mail: marco.maioli@unimore.it

${ }^{3}$ Dipartimento di Matematica, Università di Bologna, I-40127-Bologna, Italy - E.mail: martinez@dm.unibo.it
} 


\section{Introduction}

The anharmonic oscillators are interesting non-solvable models of quantum physics, as the cubic one, for their simplicity. New interest comes from the theory of the $\mathcal{P} \mathcal{T}$-symmetric operators. In particular, the interest is directed to the summability of the perturbation series, also in connection to similar problems in quantum field theory.

Many years ago [1], the Padé summability (PS) of the perturbation series of the energy levels of the quartic anharmonic oscillator with Hamiltonian $K_{4,1}(\beta)=p^{2}+x^{2}+\beta x^{4}$ was proved.

Some years later [2], the Borel summability of the perturbation series of each eigenvalue $E_{n, \alpha}(\beta), n \in \mathrm{N}$, of the cubic anharmonic oscillator,

$$
H_{\alpha}(\beta)=p^{2}+\alpha x^{2}+i \sqrt{\beta} x^{3},
$$

for a fixed $\alpha>0$, was proved. This result was later extended [3], giving the distributional Borel summability [4] of the perturbation series, in the case of negative $\beta$.

The conjecture of Bessis-Zinn Justin (BZJ), was proved by Dorey et al. [11] at $\alpha=0$. Shin [5] extended the proof to $\alpha \in \mathrm{R}$, and proved the positivity of the eigenvalues $\left\{E_{n, \alpha}(\beta)\right\}_{n}$ for $\alpha \geq 0, \beta>0$. Strangely enough, Bessis didn't suggest, as far as we know, that the reality of the eigenvalues was a consequence of his loved PS. Some years later, Bender and Weniger have given numerical evidence of PS [8].

The BZJ conjecture was later extended by Bender and Boettcher (BB) [6], to the family of $\mathcal{P} \mathcal{T}$-symmetric (PTS) Hamiltonians,

$$
H_{N, \alpha}(1)=p^{2}+\alpha x^{2}-(i x)^{N},
$$

$\alpha \geq 0, N \geq 2$, with analytic eigenfunctions $\phi(z)$, where $z=x+i y$, vanishing at infinity on the two Stokes angular sectors of the complex plane,

$$
S_{ \pm 1}^{N}=\left\{\left|\arg (i z) \pm \frac{2 \pi}{(N+2)}\right|<\frac{\pi}{(N+2)}\right\}
$$

The last conjecture was proved, as a part of a more general result, by Vladimir Bouslaev and one of us [9] (see also [10]), in the relevant case of $N=4$. Shin has proved the BB conjecture, in the general case, for $\alpha \leq 0$ [5]. From now on, we restrict the discussion to the cubic oscillator. The family of operators $H_{1}(\beta)$ is an analytic family of type A on the cut plane $C_{c}=\{\beta \in$ $\mathrm{C} ; \beta \neq 0,|\arg (\beta)=\theta|<\pi\}$, and we have the spectral equivalence [12],

$$
H_{1}(\beta) \sim \alpha^{-1 / 2} H_{\alpha}(1)
$$


where $\alpha=\exp (-i 2 \theta / 5)$. For $\beta$ at the boundary of the cut, for instance, $\beta=b \exp (-i \pi)=-b-i 0^{+}, b>0$, the mechanical problem defined by the Hamiltonian $H_{\alpha}(\beta)$, for $\alpha=a>0$, is uncomplete in both classical and quantum case and can be defined by the physical hypothesis of the disapperance of the particle when it reaches infinity. In the quantum case, this means to define the Hamiltonian by the Gamow condition at $-\infty[2]$. The eigenvalues have the meaning of resonances and the eigenfunctions have the meaning of metastable states for the dynamical problem. Thus, we expect, and we prove, a negative imaginary part of the eigenvalues, related, in the usual way, to the lifetime of the metastable state.

We consider the eigenfunction $\psi_{n, a, \beta}(z)$, for a fixed $a>0$, where $n \in \mathrm{N}$ is the number of its nodes, and $\beta$ is on the complex cut plane $C_{c}$. The $n$ nodes, numerically studied in [7] for positive $\beta$, are stable at $\beta=0$ and are the only zeros on the lower half complex plane $\mathrm{C}_{-}=\{z \in \mathrm{C} ; \Im(z)=y<0\}$. On the other side, there are no zeros on the strip $0 \leq \Im(z) \leq y_{+}=2 a \Re \sqrt{\beta} / 3 b$.

We use the Loeffel-Martin method and the complex semiclassical Sibuya picture for proving the confinement of the nodes. We use also the hypothesis of the boundedness of each eigenvalue for bounded parameters. This hypothesis is verified by the Bohr-Sommerfeld quantization rule (21)(30) and the invariance of the number of nodes. This fact forbids both, the disappearence at infinity of the perturbative eigenvalues, and the appearence of non-perturbative eigenvalues. We also prove the semiclassical nature of the eigenvalues (30).

The crossings of eigenvalues and the branch point singularities are forbidden by the unique characterization of the eigenfunctions by the number of their nodes, and the simplicity of the spectrum. Let us remember that we have the extended $\mathcal{P} \mathcal{T}$ symmetry (see [2]) of the complex Hamiltonians,

$$
H_{1}(\beta)=\mathcal{P} H_{1}^{*}(\bar{\beta}) \mathcal{P}, \text { where } \mathcal{P} \psi(x)=\psi(-x) .
$$

The isolation and analyticity of each eigenvalue on the cut plane $C_{c}$ and the unique sum of the perturbation series, imply the extended $\mathcal{P} \mathcal{T}$ symmetry of the eigenfunctions, $\psi_{n, 1, \beta}(x)=\bar{\psi}_{n, 1, \bar{\beta}}(-x)$, and eigenvalues $E_{n, 1}(\beta)=$ $\bar{E}_{n, 1}(\bar{\beta})$. The identity, obtained by complex scaling for $\beta \neq 0,|\arg (\beta)|<\pi$,

$$
\left\{E_{n, 1}(\beta)=\alpha^{-1 / 2} E_{n, \alpha}(1)\right\}_{n \in \mathrm{N}},
$$

where $\alpha=\beta^{-2 / 5}$, allows the global analytic continuation on the Riemann surface of $\beta^{1 / 5}$, of the set of the eigenvalues. In particular, we prove the power law behavior of the eigenvalues at $\beta=\infty$ by the scaling law (4) and the analyticity of $\left\{E_{n, \alpha}(1)\right\}_{n}$ at $\alpha=0$. 
We prove the PS of the perturbation series of each eigenvalue to the eigenvalue itself. In order to be more precise, let us fix $n \in \mathrm{N}=\{0,1,2, \ldots\}$, and set the simplified notations for the once subtracted eigenvalue, $f(\beta)=$ $\left(E_{n, 1}(\beta)-E_{n, 1}(0)\right) / \beta$, for any $\beta$ on the cut complex plane $C_{c}$. Thus, for $\beta \in \mathrm{C}_{c}$, we have the Stieltjes representation for $f(\beta)$, and the asymptotics for small $b=|\beta|$ given by the formal perturbation series [2],

$$
f(\beta)=\int_{0}^{\infty} \frac{1}{(1+\beta \lambda)} \rho(\lambda) d \lambda \sim \Sigma(\beta)=\sum_{k=0}^{\infty} c_{k+1} \beta^{k},
$$

where $\rho(\lambda)$ is non-negative, and the $\left\{c_{j}\right\}_{j \in \mathrm{N}}$ are the perturbation coefficients of $E_{n, 1}(\beta)$.

Thus, we prove, in a new way, the positivity of the eigenvalues, for positive $\beta$,

$E_{n, 1}(\beta)=E_{n, 1}(0)+\beta f(\beta)=E_{n, 1}(0)+\beta \int_{0}^{\infty} \frac{1}{(1+\beta \lambda)} \rho(\lambda) d \lambda \geq E_{n, 1}(0)>0$.

The PS of the perturbation series to the eigenvalue is defined by the limit,

$$
f(\beta)=\lim _{k \rightarrow \infty} R_{k}^{k}(\beta)
$$

where the $R_{k}^{k}(\beta)=P_{k}(\beta) / Q_{k}(\beta)$, are the diagonal Padé approximants, $P_{k}(\beta)$, $Q_{k}(\beta)$ are polynomials of order $k$, with $Q_{k}(0)=1$, completely defined by the asymptotics for $|\beta|$ small,

$$
\left|R_{k}^{k}(\beta)-\Sigma^{2 k+1}(\beta)\right|=O\left(|\beta|^{2 k+1}\right),
$$

where $\Sigma^{k}(\beta)=\sum_{j=0}^{k-1} c_{j+1} \beta^{j}$.

The semiclassical behavior, for large positive $\lambda$, of the discontinuity,

$$
\ln (\rho(\lambda))=-C^{-1} \lambda(1+O(\ln (\lambda) / \lambda)),
$$

where $C=15 / 8$, agrees with the asymptotics of the perturbation coefficients for large $j$, as computed in [8], for $n=0$,

$$
c_{j}=(-1)^{j+1} 4 \sqrt{15} C^{j}(2 \pi)^{-3 / 2} \Gamma(j+1 / 2)(1+O(1 / j)) .
$$

For numerical aspects, as the interesting similarity of this perturbation series with the one of the quartic anharmonic oscillator, see reference [8].

In Section 2 we discuss the operators for $\beta$ at the boundaries of the complex cut plane. In Section 3 we consider the stability, analyticity and asymptotics 
of the eigenvalues and the nodes of the eigenfunctions for small $|\beta|$. In Section 4 we confine the nodes on the lower complex half plane and we extends the results of Section 2. In Section 5 we prove the stability of the nodes for small parameter. In Section 6 we prove the stability of the nodes for large parameter. In Section 7 we prove the boundedness of an eigenvalue for bounded parameters. In Section 8 we prove the absence of non-perturbative eigenvalues. In Section 9 we prove the power law behavior at infinite parameter. In Section 10 we prove the Padé summability of the perturbation series.

\section{The imaginary part of the eigenvalues on the cut: a preliminary study}

Let $\beta=b \exp (i \theta), b>0$, the family of operators $H_{1}(\beta)$ is an analytic family of type $\mathrm{A}$ on the cut plane $C_{c}=\{\beta \in \mathrm{C} ; \beta \neq 0,|\arg (\beta)|<\pi\}$, and we have the spectral equivalence (see [2])

$$
H_{1}(\beta)=\mathcal{P} H_{1}^{*}(\bar{\beta}) \mathcal{P} \sim \alpha^{-1 / 2} H_{\alpha}(b),
$$

where $\alpha=\exp (-i 2 \theta / 5)$ and $\mathcal{P} \psi(x)=\psi(-x)$. The identity of the sets of eigenvalues,

$$
\left\{E_{n, 1}(\beta)\right\}_{n \in \mathrm{N}}=\alpha^{-1 / 2}\left\{E_{n, \alpha}(b)\right\}_{n \in \mathrm{N}},
$$

defines the global analytic continuation from $C_{c}$ to all the Riemann surface of $\beta^{1 / 5}$, of the set of the eigenvalues, $\left.\left\{E_{n, 1}(\beta)\right)\right\}_{n \in \mathrm{N}}$.

In particular, we are interested here in the eigenvalues $\left\{E_{n, 1}(\beta)\right\}_{n \in \mathrm{N}}$ for $\beta$ on the closed cut plane $\bar{C}_{c}=\{\beta \in \mathrm{C} ; \beta \neq 0,|\arg (\beta)| \leq \pi\}$.

The operators $H_{1}(\beta)$ on the borders of the cut, $\arg (\beta)=\theta= \pm \pi$, are uncomplete, and they are defined by the choice of the fundamental behavior at $\pm \infty$ respectively [2]. This choice is fixed by the spectral equivalence (3).

In the case of $\theta=-\pi, i \sqrt{\beta}$ positive, the Hamiltonian is real, but both the classical problem and the quantum one, are uncomplete. In the quantum case, all the solutions are $L^{2}$ at $-\infty$. The choice of the Gamow behavior (corresponding to negative current density) at $-\infty$, gives eigenvalues with the meaning of resonances and eigenstates with the meaning of metastable states.

In the other case, for $\theta=\pi, i \sqrt{\beta}$ negative, with the choice of the anti-Gamow behavior at $+\infty$, we have the correct definition of the operator and we verify the spectral equivalence, $H_{1}(\beta) \sim \sqrt{\alpha} H_{\alpha}(b), \alpha=\exp (-i 2 \pi / 5)$. 
Actually, we have the following behavior of the eigenfunction $\psi_{n, 1, \beta}$ for $x$ large:

$$
\psi_{n, 1, \beta}(x) \sim \frac{K}{x^{3 / 4}} \exp \left(-\sqrt{i} \sqrt[4]{\beta} x^{5 / 2}\right)=\frac{K}{x^{3 / 4}} \exp \left(-i \sqrt[4]{b} x^{5 / 2}\right)
$$

The same behavior is defined by the scaled eigenfunction, satisfying the $L^{2}$ condition,

$$
\psi_{n, \alpha}(b)(x) \sim \frac{K_{1}}{x^{3 / 4}} \exp \left(-\sqrt{i} \sqrt[4]{b} x^{5 / 2}\right) \rightarrow 0
$$

as $x \rightarrow \infty$.

Now, let us consider the translated Hamiltonian

$$
T_{-\epsilon} H(1, \beta) T_{-\epsilon}^{-1}=H_{-\epsilon, 1}(\beta),
$$

where the complex translation, is defined by $T_{-\epsilon} \psi(x)=\psi_{-\epsilon}(x)=\psi(x-i \epsilon)$, for $\epsilon>0$. Because of the translation analyticity of the Hamiltonian, we have the spectral equivalence,

$$
H_{-\epsilon, 1}(\beta) \sim H_{1}(\beta)
$$

for $\beta \in \dot{C}_{c}$. This equivalence can be extended to the case of $\theta=\pi$, if the operator $H_{1}(\beta)$ is defined, as above, by the correct condition at $+\infty$. Let us consider an eigenfunction $\psi_{-\epsilon, n, 1, \beta}(x)=\psi_{n, 1, \beta}(x-i \epsilon)$ of $H_{-\epsilon, 1}(\beta)$, for $\beta=b \exp (i \pi) \neq 0$. We have the $L^{2}$ behavior,

$$
\psi_{n, 1, \beta}(x-i \epsilon) \sim \frac{K_{2}}{x^{3 / 4}} \exp \left(-i \sqrt[4]{b} x^{5 / 2}(1-5 i \epsilon / x)\right) \rightarrow 0,
$$

as $x \rightarrow+\infty$, if $\psi_{n, 1, \beta}$ has the anti-Gamow behavior.

Let us consider the numerical range of the translated operator, for $\beta=$ $b \exp (i \pi)$,

$$
H_{-\epsilon, 1}(\beta)=p^{2}+V_{1, \beta}(x-i \epsilon),
$$

for $\epsilon>0$, where $V_{1, \beta}(x-i \epsilon)=(x-i \epsilon)^{2}-\sqrt{b}(x-i \epsilon)^{3}$ and

$$
\Im V(x-i \epsilon)=-\epsilon\left(2 x-\sqrt{b}\left(3 x^{2}-\epsilon^{2}\right)\right) \geq-\frac{\epsilon}{3 \sqrt{b}}\left(1+3 b \epsilon^{2}\right) .
$$

Thus, the intersection of the numerical ranges of the operators $\left\{H_{-\epsilon, 1}(\beta)\right\}_{\epsilon}$, for all $\epsilon>0$, is contained on $\overline{\mathrm{C}}_{+}=\{z \in \mathrm{C}: \Im z \geq 0\}$. Thus, we have "antiresonances" $E_{n}(1, b \exp (i \pi))=E_{n}\left(1,-b+i 0^{+}\right)$, with $\Im E_{n}\left(1,-b-i 0^{+}\right) \geq 0$, as the usual anti-resonances [16].

In a similar way, we prove that $\Im E_{n, 1}\left(-b-i 0^{+}\right) \leq 0$. Thus, we have:

Lemma 1. An eigenvalue $E(\beta)=E_{n, 1}(\beta), n \in \mathrm{N}, \beta=b \exp (i \pi)=-b+i 0^{+}$, 
$b>0$, of $H_{1}(\beta) \sim \alpha^{-1 / 2} H_{\alpha}(b), \alpha=\exp (-2 i \pi / 5)$, has a non-negative imaginary part: $\Im E(b \exp (i \pi)) \geq 0$. On the other side, for $\beta=b \exp (-i \pi)=$ $-b-i 0^{+}, b>0$, we have $\Im E(b \exp (-i \pi)) \leq 0$.

\section{Analyticity, symmetry and stability of the nodes for small parameter}

Let us consider the analytic family of type A of compact resolvent operators,

$$
H_{\alpha}(\beta),
$$

on the domain $D=D\left(p^{2}\right) \cap D\left(x^{3}\right)$ for fixed $\alpha \in \mathrm{C}, \beta$ on the cut plane

$$
\mathrm{C}_{c}=\{\beta \in \mathrm{C} ; b=|\beta|>0,|\arg (\beta)=\theta|<\pi,\}
$$

([2], Theorem 2.9).

We fix, for example, $\alpha=1$.

We remember that $H_{1}(\beta)$, for $\beta$ on the borders of $\mathrm{C}_{c}$, for instance at $\arg (\beta)=$ $\pi$ (the other case, $\arg (\beta)=-\pi$, is similar), is defined by the Gamow condition at $-\infty$.

The eigenvalue $E_{n, 1}(\beta)$, for a fixed $n=0,1, \ldots$, of $H_{1}(\beta)$ is an eigenvalue also of the operator $\alpha^{1 / 2} H_{\alpha}(b), E_{n, 1}(\beta)=\alpha^{1 / 2} E_{n, \alpha}(b)$, (the index $n$ is related to the number of nodes of the eigenfunction) where

$$
\alpha=(b / \beta)^{2 / 5}=\exp (-2 i \theta / 5) .
$$

In particular $E_{n, 1}(b \exp ( \pm \pi))=\sqrt{\alpha} E_{n, \alpha}(b)$, where $\alpha=\exp (\mp 2 i \pi / 5)$.

Moreover, the eigenvalue $E_{n, 1}(\beta)$ of $H_{1}(\beta)(p, x)$, for $\beta=b \exp ( \pm i \pi)$, is also an eigenvalue of the translated operator,

$$
H_{1}(\beta)(p, x \pm i \epsilon)=p^{2}+(x \pm i \epsilon)^{2}+i \sqrt{\beta}(x \pm i \epsilon)^{3},
$$

for $\epsilon>0$.

For $\beta$ in the completed cut plane, $\overline{\mathrm{C}}_{c}=\{\beta \in \mathrm{C} ; \beta \neq 0,|\arg (b)| \leq \pi$, $\}$ we have the spectral equivalence by scaling:

$$
H(\alpha, b) \sim(\alpha)^{-1 / 2} H(1, \beta)
$$

where $\alpha=(b / \beta)^{2 / 5}=\exp (-2 i \theta / 5)$ with $|\arg (\alpha)|<\pi / 2$.

In place of the limit of $H_{1}(\beta)$, as $\beta \rightarrow 0$, we consider the norm resolvent limit 
$H_{\alpha}(b) \rightarrow H_{\alpha}(0)$, for $\alpha=(b / \beta)^{2 / 5}$ fixed, as $b \rightarrow 0$. Let us notice that $H(\alpha, 0)$, for $\alpha \neq 0$, is defined on the domain $D=D\left(p^{2}\right) \cap D\left(x^{2}\right)$ (see Theorem 2.13 on reference [2], and its extension on reference [3]).

We have the result of strong asymptotism of the eigenvalues:

\section{Theorem 1.}

For $n=0,1, \ldots$, let $E_{n, 1}(\beta)$ be an eigenvalue, and let $c_{k}, k \in \mathrm{N}$, be its perturbation coefficients,

$$
f(\beta)=\frac{\left(E_{n, 1}(\beta)-E_{n, 1}(0)\right)}{\beta} .
$$

Then, there exists $b_{n}>0$ such that $f(\beta)$ is analytic on the bounded sector,

$$
\Omega_{n}=\left\{\beta \in \mathrm{C} ; 0<|\beta|<b_{n},|\arg (\beta)| \leq \pi\right\},
$$

and there exist numbers $A, C>0$, such that

$$
\left|f(\beta)-\Sigma^{N}(\beta)\right|<A C^{N} N !|\beta|^{N},
$$

where $\Sigma^{N}(\beta)=\sum_{k=0}^{N-1} c_{k+1}(\beta)^{k}$, uniformly for $N-1 \in$ $N$ and $\beta \in \Omega_{n}$.

Proof See reference [2], Theorem 3.2., (where $\beta$ is our $i \sqrt{\beta}$ ), extended in reference [3] (for $k=1)$.

\section{Remark 1: the stability of the nodes.}

Together with the stability of the eigenvalues, we have the stability of the eigenfunctions. In particular, we are interested in the stability of their zeros, or nodes.

We have the limit of the eigenvalue $E_{n}(1, \beta) \rightarrow E_{n}(1,0)$ and the strong limit of the eigenvector $\psi_{n, 1, \beta} \rightarrow \psi_{n, 1,0}$ as $b=|\beta| \rightarrow 0^{+}$, for $\beta \in \Omega_{n}$, $\arg (\beta)$ fixed, $|\arg (\beta)| \leq \pi$. Thus we have the limit $\psi_{n, 1, \beta}(z) \rightarrow \psi_{n, 1,0}(z)$ as $b=|\beta| \rightarrow 0^{+}$, for $\beta \in \Omega_{n}, \arg (\beta)$ fixed, $|\arg (\beta)| \leq \pi$, uniformly for $z$ on a compact of the complex plane.

Since the perturbed eigenfunctions are entire, as the unperturbed ones, we have the stability of the $n$ zeros of $\psi_{n, 1,0}(z)$ for $b=|\beta|$ small.

For any fixed regular closed curve $\gamma=\partial \Gamma$ on the complex plane, oriented in the positive sense, around the segment of extremes $\left(x_{-}, x_{+}\right)$, where $x_{ \pm}=$ $\pm \sqrt{E_{n}(1,0)}= \pm \sqrt{(2 n+1)}$, we have the constant number of zeros (nodes) in $\Omega_{n}$ :

$$
n=\frac{1}{2 i \pi} \oint_{\gamma} \frac{\psi_{n, 1, \beta}^{\prime}(z)}{\psi_{n, 1, \beta}(z)} d z=\frac{1}{2 i \pi} \oint_{\gamma} \frac{\psi_{n, 1,0}^{\prime}(z)}{\psi_{n, 1,0}(z)} d z,
$$


for $\beta \in \Omega_{n}^{\prime}$, where

$$
\Omega_{n}^{\prime}=\left\{\beta \in \mathrm{C} ; 0<|\beta| \leq b_{n}^{\prime},|\arg (\beta)| \leq \pi\right\},
$$

and $0<b_{n}^{\prime} \leq b_{n}$.

Let us set $\psi_{\beta}=\psi_{n, 1, \beta}$ and $\psi_{0}=\psi_{n, 1,0}$ and apply the theorem of Rouché [17]. Since the zeros of $\psi_{0}(z)$ are not on $\gamma$, there exists $M>0$, such that $\left|\psi_{0}\right| \geq M>0$ uniformly on $\gamma$. Moreover, $\left|\psi_{\beta}(z)-\psi_{0}(z)\right| \rightarrow 0$ uniformly for $z$ on the compact $\gamma$, because of the analyticity. Thus, we have $\left|\psi_{0}(z)\right|>\left|\psi_{\beta}(z)-\psi_{0}(z)\right|$ for $z$ on $\gamma$ and for $\beta \in \Omega_{n}^{\prime} \bigcup\{0\}$, so that the Rouché theorem applies.

We shall see (Theorem 2) that, for $\beta \in \Omega_{n}^{\prime}$, the $n$ zeros (nodes) are confined on $\Gamma \cap \mathrm{C}_{-}$, where $\mathrm{C}_{-}=\{z \in \mathrm{C} ; \Im(z)<0\}$. The $n$ nodes are the only zeros of $\psi(z)$ on $\mathrm{C}_{-}$.

\section{Absence of zeros of the eigenfunctions on the real axis, and above it}

We consider the operator

$$
H(a, \beta)=p^{2}+a x^{2}+i \sqrt{\beta} x^{3},
$$

and the eigenvalue $E_{n, a}(\beta)$, with eigenfunction $\psi_{n, a, \beta}$, where $n=0,1, \ldots$, $a \geq 0,|\beta|=b>0, \theta=\arg (\beta),|\theta| \leq \pi$.

Let us fix $|\theta|<\pi, a>0$.

We call $z=x+i y$ the $x$ variable extended to the complex plane. We consider the eigenvalues $E=E_{n, a}(\beta)$, and eigenfunctions $\psi_{E}(z)=\psi_{n, a, \beta}(z)$, where the label $n$ is related to the number of zeros stable at $\beta=0$.

On the strip

$$
A(a, \beta)=\left\{z \in \mathrm{C} ; 0 \leq \Im(z) \leq y_{+}=\frac{2 a \Re \sqrt{\beta}}{3 b}=\frac{2 a}{3 \sqrt{b}} \cos \left(\frac{\theta}{2}\right)\right\},
$$

there are no zeros of the eigenfunction $\psi_{n, a, \beta}(z)$.

Theorem 2. On the strip

$$
A(a, \beta)=\left\{z \in \mathrm{C} ; 0 \leq \Im(z) \leq y_{+}=\frac{2 a \Re \sqrt{\beta}}{3 b}=\frac{2 a}{3 \sqrt{b}} \cos \left(\frac{\theta}{2}\right)\right\},
$$


there are no zeros of any eigenfunction $\psi_{E}(z)$ of $H(a, \beta)$, with eigenvalue $E$, where $a>0$, and $b=|\beta|>0, \theta=\arg (\beta),|\theta| \leq \pi$.

Proof.

Let us, at first, set $|\theta|<\pi$, and consider the translated operator $H_{a, \beta, y}=$ $p^{2}+V_{y}$, where

$$
\begin{gathered}
V_{y}=V_{y}(x)=a(x+i y)^{2}+i \sqrt{\beta}(x+i y)^{3}= \\
=a x^{2}-a y^{2}-3 \sqrt{\beta} y x^{2}+\sqrt{\beta} y^{3}+2 a i y x-3 i \sqrt{\beta} y^{2} x+i \sqrt{\beta} x^{3} .
\end{gathered}
$$

Let $\psi_{y}(x)=\psi_{E}(x+i y)$. We have:

$$
\psi_{E}(x+i y)=\psi_{y}(x) \neq 0
$$

for every $x \in \mathrm{R}$, for $0 \leq y \leq y_{+}=2 a \Re(\sqrt{\beta}) / 3 b$.

For $0 \leq y \leq y_{+}$,

$$
-\Im\left(\psi_{y}(r) \frac{d \overline{\psi_{y}(r)}}{d r}\right)=\int_{r}^{\infty} \Im\left(V_{y}(x)-E\right)\left|\psi_{y}(x)\right|^{2} d x>0
$$

or

$$
-\Im\left(\psi_{y}(r) \frac{d \overline{\psi_{y}(r)}}{d r}\right)=-\int_{-\infty}^{r} \Im\left(V_{y}(x)-E\right)\left|\psi_{y}(x)\right|^{2} d x>0
$$

for any $r \in \mathrm{R}$.

The proof is based on the monotonicity of

$$
f(x)=\Im\left(V_{y}(x)-E\right)=R\left(x^{3}-3 y^{2} x\right)+2 a x y-3 I y x^{2}+c,
$$

where $R=\Re(\sqrt{\beta}), I=\Im(\sqrt{\beta})$ and $c$ is a constant, that is, the non-negativity of $f^{\prime}(x)$,

$$
f^{\prime}(x)=\Im\left(V_{y}(x)-E\right)^{\prime}=3 R x^{2}-6 I y x-3 R y^{2}+2 a y=A x^{2}+B x+C \geq 0,
$$

where: $A=3 R, B=-6 I y, C=-3 R y^{2}+2 a y$. We impose the non-positivity of the discriminant:

$$
\left(B^{2}-4 A C\right) / 4=12 y[3 b y-2 R a] \leq 0,
$$

proved for

$$
0 \leq y \leq y_{+}=\frac{2 R a}{3 b}=\frac{2 a}{3 \sqrt{b}} \cos \left(\frac{\theta}{2}\right)
$$


We have absence of zeros for $0 \leq \Im z \leq y_{+}$.

In the case of $|\arg (\beta)|=\pi$, we have the limits of the equations (12)(13), and for any $r \in \mathrm{R}$,

$$
\begin{gathered}
-\Im\left(\psi(r) \frac{d \overline{\psi(r)}}{d r}\right)=\int_{r}^{\infty} \Im(V(x)-E)\left|\psi_{E}(x)\right|^{2} d x=-\int_{r}^{\infty} \Im(E)\left|\psi_{E}(x)\right|^{2} d x>0 \\
\quad \text { or } \int_{-\infty}^{r} \Im(E)\left|\psi_{E}(x)\right|^{2} d x>0
\end{gathered}
$$

if $\Im(E) \neq 0$. Thus, the nodes have negative imaginary part, if the imaginary part of the eigenvalue is different from zero.

Proof that the imaginary part of the eigenvalues is non zero.

For $b=|\beta|, \arg (\beta)=-\pi$ fixed and $-r$ large, the normalized wave function $\psi_{E}, \quad\left|\psi_{E}\right| \mid=1$, satisfies the Gamow condition. This means that the eigenfunction is proportional to the Gamow solution $\psi_{E}=c_{-} \psi_{-}$, with $c_{-} \neq 0$, if $\psi_{E} \neq 0$. The Gamow solution is defined by $\psi_{-}^{\prime}(r) / \psi_{-}(r) \rightarrow-i$ and a normalization satisfying $\sqrt{-V(r)}\left|\psi_{-}(r)\right|^{2} \rightarrow 1$, as $r \rightarrow-\infty$. Thus, we have,

$$
\begin{gathered}
-\Im(E)=-\frac{1}{\int_{r}^{+\infty}\left|\psi_{E}(x)\right|^{2} d x} \Im\left(\left|\psi_{E}(r)\right|^{2} \frac{\psi_{E}^{\prime}(r)}{\psi_{E}(r)}\right) \sim \\
\sim \frac{1}{\int_{r}^{+\infty}\left|\psi_{E}(x)\right|^{2} d x} \sqrt{-V(r)}\left|\psi_{E}(r)\right|^{2} \rightarrow\left|c_{-}\right|^{2}>0,
\end{gathered}
$$

as $r \rightarrow-\infty$, implying $\Im(E)<0$.

In the general case, the nodes stay on the half plane: $C_{-}=\{z \in \mathrm{C} ; \Im(z)<$ $0\}$. Thus, we have:

Lemma 1'. An eigenvalue $E(\beta)=E_{n, 1}(\beta), n \in \mathrm{N}, \beta=b \exp (i \pi)=$ $-b+i 0^{+}, b>0$, of $H_{1}(\beta) \sim \alpha^{-1 / 2} H_{\alpha}(b), \alpha=\exp (-2 i \pi / 5)$, has a nonnegative imaginary part: $\Im E(b \exp (i \pi))>0$. On the other side, for $\beta=$ $b \exp (-i \pi)=-b-i 0^{+}, b>0$, we have $\Im E(b \exp (-i \pi))<0$.

\section{The semiclassical confinement of the nodes for small parameter}

We prove the absence, for $|\beta|>0$ small, $|\arg (\beta)| \leq \pi$, of any zero of the eigenfunction $\psi_{\beta}(z)=\psi_{n, 1, \beta}(z)$ of $H_{1}(\beta)$, with eigenvalue $E_{\beta}=E_{n, 1}(\beta)$, for 
a fixed $n=0,1, \ldots$, on the lower half plane, $z \in \mathrm{C}_{-}$, for large $|z|$.

Let us consider the semiclassical quantity,

$$
p_{\beta}(z)=\sqrt{V_{\beta}(z)-E_{\beta}},
$$

where $V_{\beta}(z)=z^{2}+i \sqrt{\beta} z^{3}$ and $E_{\beta}=E_{n, 1}(\beta)$ is the eigenvalue of the Hamiltonian with eigenfunction $\psi_{\beta}(z)$. There are three zeros of $p_{\beta}(x)$. Two zeros $z_{ \pm}(\beta)$, converge $z_{ \pm}(\beta) \rightarrow z_{ \pm}= \pm \sqrt{E_{0}}$ as $\beta \rightarrow 0$, in the sector $|\arg (\beta)|<\pi$. The third one, $z_{0}(\beta)$ diverges, $z_{0}(\beta) \sim i / \sqrt{\beta}$, as $\beta \rightarrow 0$, in the sector $|\arg (\beta)|<\pi$.

Let $n=0,1, .$. fixed, $z \in \mathrm{C}_{-},|z|>>\left|z_{ \pm}\right|$, and $\beta \in \Omega_{n}^{\prime}$, we define:

$$
f(\beta, z)=f(n, 1, \beta, z)=\frac{\left|\psi_{\beta}^{\prime}(z)\right|}{\left|p_{\beta}(z) \psi_{\beta}(z)\right|},
$$

where $p_{\beta}(z)$ is defined above (16). We have,

$$
f(\beta, z) \rightarrow 1
$$

for $|z| \rightarrow \infty$, uniformly for $z$ on the sector $|\arg (i z)| \leq \pi / 2-\epsilon$, for any $\epsilon$ $0<\epsilon<\pi / 2,0 \leq|\beta| \leq b_{n}^{\prime}$, for fixed $\arg \beta,|\arg (\beta)|<\pi$. This means that no node of $\psi_{\beta}(z)$ goes to (or comes from) infinity on the sector $|\arg (i z)| \leq \pi / 2$, for this set of parameters.

\section{Theorem 3.}

Let $\psi_{\beta}(z)=\psi_{n, 1, \beta}(z)$ be an eigenfunction with $n$ nodes and eigenvalue $E=$ $E_{n}$.

No one of its nodes goes to (or come from) infinity on the sector $|\arg (i z)|<$ $\pi / 2$, for $|\beta| \leq b_{n}^{\prime},|\arg (\beta)|<\pi$.

\section{Remark 2.}

We can extend the limit (18) uniformly for $0 \leq b=|\beta| \leq b_{n}^{\prime}$ fixed, and $|\arg \beta| \leq \pi$. Thus we extend the barrier for the zeros at infinity on the sector $|\arg (i z)| \leq \pi / 2$, to the full $\beta$-sector

$$
\bar{\Omega}_{n}^{\prime}=\left\{\beta \in \mathrm{C} ; 0 \leq|\beta| \leq b_{n}^{\prime},|\arg (\beta)| \leq \pi\right\},
$$




\section{The semiclassical confinement of the nodes for large parameter}

Let the Hamiltonian be $H_{a}=p^{2}+V_{a}=H_{a}(\beta)=p^{2}+V_{a}(\beta)$, and $E=E_{n, a}(\beta)$ for fixed $n=0,1, .$. , be an eigenvalue with eigenfunction $\psi_{E}(z)=\psi_{n, a, \beta}(z)$ for fixed $\beta,|\beta| \geq b_{n}^{\prime},|\arg (\beta)| \leq \pi$, and $0 \leq a \leq 1$. We have $E \neq 0$, $|\arg (E)| \leq \pi / 2$, because of the numerical range.

Now, we make the hypothesis of boundendness of an eigenvalue for the parameters restricted on a compact. This allows to prove the stability of the nodes of its eigenfunction. We will prove later that the eigenvalue is bounded if the number of nodes is stable.

\section{Hypothesis I.}

The eigenvalue $E=E_{n, a}(\beta)$ for fixed $n=0,1, . ., \beta,|\beta|=b_{n}^{\prime}>0$, where $b_{n}^{\prime}$ is given in Theorem 1, is uniformly bounded for $|\arg (\beta)| \leq \pi$, and $0 \leq a \leq 1$.

Let us recall [13] the 5 Stokes angular sectors of the complex $x$ plane, for $\beta \neq 0$,

$$
S_{k}=S_{k}(\arg (\beta))=\left\{z \in \mathrm{C} ;\left|\arg (i z)+\frac{1}{10} \arg (\beta)-\frac{2 k \pi}{5}\right|<\frac{\pi}{5}\right\},
$$

$-2 \leq k \leq 2$.

The eigenfunction $\psi_{E}(z)$ is an entire function and,

$$
\left(\psi_{E}(z), \psi_{E}^{\prime}(z)\right) \rightarrow 0
$$

as $|z| \rightarrow \infty$, for $\arg (z)$ in each one of the two Stokes angular sectors $S_{ \pm 1}$. On the other side, $\psi_{E}(z)$ is purely divergent in the other three sectors $S_{0}, S_{ \pm 2}$, and has no zeros [13] in the full angular sector of the complex plane

$S=S(\arg (\beta))=S_{-2} \bigcup \bar{S}_{-1} \bigcup S_{0} \bigcup \bar{S}_{1} \bigcup S_{2}=\left\{x \in \mathrm{C} ;\left|\arg (i z)+\frac{1}{10} \arg (\beta)\right|<\pi\right\}$

for large $|z|$.

We have the following result.

\section{Theorem 3'.}

Let $\beta$ be fixed, with $|\beta|=b_{n}^{\prime}>0,|\arg (\beta)| \leq \pi$, and $0 \leq a \leq 1, \psi_{a}(z)=$ $\psi_{n, a, \beta}(z)$ be an eigenfunction, with eigenvalue $E=E_{a}=E_{n, a}(\beta)$, where the index $n=0,1, .$. , is the number of its nodes at $a=1$.

Moreover, we assume the Hypothesis I.

Then, no one of its nodes goes to (or comes from) infinity on the sector

$$
S_{-}=\{z \in \mathrm{C} ;|\arg (i z)| \leq \pi / 2\} .
$$




\section{Remark 3.}

Considering also Theorem 2, we have the invariance of the number of nodes. Proof of the Theorem 3'.

Let us consider the function

$$
f_{a}(z)=\frac{\left|\psi_{a}^{\prime}(z)\right|}{\left|p_{a}(z) \psi_{a}(z)\right|}
$$

where $p_{a}(z)=\sqrt{V_{a}(z)-E_{n, a}} \sim \sqrt[4]{\beta}(i z)^{3 / 2}$ for large $|z|$ with $a, E$ fixed.

Since $\psi_{a}(z)$ is the analytic solution of the Schrödinger equation, with energy $E_{n, a}$, the zeros of $\psi_{a}(z)$ are simple and $f_{a}(z)$ has a pole where $\psi_{a}(z)$ has a zero. We have,

$$
f_{a}(z) \rightarrow 1
$$

as $|z| \rightarrow \infty$, uniformly for $|\arg (i z)| \leq \pi / 2, a \in[0,1], E$ on a compact set. This means that no zero of $\psi_{a}(z)$ goes to (or comes from) infinity on the sector $|\arg (i z)| \leq \pi / 2$, for this set of parameters.

\section{Boundedness of the eigenvalues}

We prove the boundedness of the eigenvalues $E_{n, a}(\beta)$ for bounded parameters $(n, a, \beta)$. In particular, $n=0,1, \ldots$ is fixed, $a \in\left[0, a_{n}\right]$, where $a_{n}=$ $\left(1 / b_{n}^{\prime}\right)^{2 / 5}>0, \beta \in \mathrm{C},|\beta|=1,|\arg (\beta)=\theta| \leq \pi$.

This results forbids the desappearence or appearence of eigenvalues at infinity for $\beta \neq 0$. It is better to use the following scaling:

$$
E_{n, \alpha}(1)=\exp (-i \theta / 5) E_{n, a}(\beta),
$$

where $\alpha=a \exp (-i 2 \theta / 5)$.

Thus, we should prove the boundedness of $E_{n, \alpha}(1)$ for $|\alpha| \in\left[0, a_{n}\right]$, and $|\arg (\alpha)| \leq 2 \pi / 5$ for $\alpha \neq 0$.

For our non self-adjoint operators, we use an argument slightly different from the one of the reference [1]. We directly use the semiclassical quantization and the stability of the nodes.

Theorem 4.

For any $n=0,1, . ., \alpha_{0}=0$, or $\alpha_{0} \neq 0$ with $\left|\arg \left(\alpha_{0}\right)\right| \leq 2 \pi / 5, E_{n, \alpha}(1)$ is bounded and continuous at $\alpha=\alpha_{0}$.

Proof. 
Let us consider the three parameter operators,

$$
H(\hbar, \alpha, \beta)=-\hbar^{2} p^{2}+\alpha x^{2}+i \sqrt{\beta} x^{3},
$$

and the eigenvalues $E_{n}(\hbar, \alpha, \beta)$ for $n=0,1, \ldots$. We have the spectral equivalence for real scaling:

$$
H(1, \alpha, 1) \sim H\left(\lambda^{-2}, \lambda^{2} \alpha, \lambda^{3}\right)
$$

so that

$$
E_{n}(1, \alpha, 1)=E_{n}\left(\lambda^{-2}, \lambda^{2} \alpha, \lambda^{3}\right),
$$

for $n=0,1, . . \lambda \neq 0$.

Because of the analyticity of the family of operators $H_{\alpha}(1)=H(1, \alpha, 1)$, boundedness implies continuity.

We prove the boundedness by absurd.

Let us fix $n=0,1, .$. , and $\alpha_{0}, 0 \leq a_{0}=\left|\alpha_{0}\right| \leq a_{n},\left|\arg \left(\alpha_{0}\right)\right| \leq 2 \pi / 5$ for $a_{0}<0$, and suppose $\left|E_{n}(1, \alpha, 1)\right| \rightarrow \infty$ as $\alpha \rightarrow \alpha_{0}$. For $\alpha$ near $\alpha_{0}$, we scale the Hamiltonian and use the identity:

$$
\lambda^{6 / 5} E_{n}(1, \alpha, 1)=E_{n}\left(\hbar, \alpha^{\prime}, 1\right):=\epsilon=\exp (i \theta),
$$

where $\epsilon=\epsilon(\alpha), \lambda=\hbar=\left|E_{n}(1, \alpha, 1)\right|^{-5 / 6}>0, \alpha^{\prime}=\lambda^{2 / 5} \alpha$ and $|\theta| \leq \pi / 2$. We set $\epsilon_{0}=\epsilon\left(\alpha_{0}\right) \neq 0$.

Thus, we study the semiclassical eigenvalue problem $H\left(\hbar, \alpha^{\prime}, 1\right) \psi_{n}=\epsilon \psi_{n}$, by the Bohr-Sommerfeld quantization rule. For small $\hbar$, we have,

$$
n=\frac{1}{2 i \pi} \oint_{\gamma} \frac{\psi_{n}^{\prime}(z)}{\psi_{n}(z)} d z=i \frac{1}{2 \pi \hbar} \oint_{\gamma} \sqrt{V(z)-\epsilon} d z-\frac{1}{2}+O(|\hbar|)
$$

where $n$ is the number of nodes, is obtained by the WKB approximation,

$$
\frac{\psi_{n}^{\prime}(z)}{\psi_{n}(z)}=-\frac{1}{\hbar} \sqrt{V(z)-\epsilon}-\frac{1}{4} \frac{V(z)^{\prime}}{V(z)-\epsilon}+O(|\hbar|) .
$$

Thus, the Bohr-Sommerfeld quantization reads,

$$
J(\alpha)=i \oint_{\gamma} \sqrt{V(z)-\epsilon} d z=\pi(2 n+1) \hbar+O\left(|\hbar|^{2}\right),
$$

where the phase of $\sqrt{V(z)-\epsilon}$ is defined on the sector $S_{1}$, such that it vanishes as $|z| \rightarrow \infty$, with $\arg (z)=-\pi / 6$.

Thus, the $n$ nodes are confined on the fixed compact domain $\Omega$ bounded by the regular curve $\partial \Omega=\gamma_{1} \cup \gamma_{2}$, where $\gamma_{1}$ is the arc of circle $\gamma_{1}=\{|z|=$ 
$\left.R ;|\arg (i z)| \leq(\pi / 2)+\theta^{\prime}\right\}$, (see Theorems 3-4) and $\gamma_{2}$ the segment of extrems $\left(-R \exp \left(i \theta^{\prime}\right), R \exp \left(i \theta^{\prime}\right)\right.$ ), where $\theta^{\prime}=\arg (\alpha) / 4$ (see Theorem 2). The limit $\alpha \rightarrow \alpha_{0}$ implies $\epsilon \rightarrow \epsilon_{0}, \alpha^{\prime}, \hbar \rightarrow 0$, and, for the classical action,

$$
\begin{gathered}
J(\alpha) \rightarrow J\left(\alpha_{0}\right)=i \int_{\gamma} \sqrt{i z^{3}-\epsilon_{0}} d z=\epsilon_{0}^{5 / 6} i \int_{\gamma} \sqrt{i y^{3}-1} d y= \\
=\epsilon_{0}^{5 / 6} \int_{\gamma} \sqrt{1-i y^{3}} d y=\epsilon_{0}^{5 / 6} 2 \Re\left(2 \exp (-i \pi / 6) \int_{0}^{1} \sqrt{1-x^{3}} d x\right)= \\
=\epsilon_{0}^{5 / 6} 4 \sin \left(\frac{\pi}{3}\right) \int_{0}^{1} \sqrt{1-x^{3}} d x=\epsilon_{0}^{5 / 6} 2 \sqrt{\pi} \sin \left(\frac{\pi}{3}\right) \frac{\Gamma(1+(1 / 3))}{\Gamma((1 / 3)+(3 / 2))} \neq 0
\end{gathered}
$$

where $y=z \epsilon_{0}^{-2 / 3}$, and where the phase of $\sqrt{i z^{3}-\epsilon_{0}}$ vanishes as $|z| \rightarrow \infty$, for $\arg (z)=-\pi / 6$, and where $\gamma$, in this semiclassical approximation, has been distorted to a regular path encircling the origin and both the turning points $z_{ \pm}$.

As a result, for the left hand of equation (21) we have,

$$
J(\alpha) \rightarrow 0
$$

as $\alpha \rightarrow \alpha_{0}, \hbar \rightarrow 0$, in contradiction with the limit of the left hand of equation (21), as written in equation (22). The proof is similar for $\alpha_{0}=0$.

Let us notice that the same analysis gives the correct semiclassical behavior of the eigenvalues [6], [5], for large $n$. From the equations (22) and (21), we have,

$$
\epsilon_{0}^{5 / 6} 2 \sqrt{\pi} \sin \left(\frac{\pi}{3}\right) \frac{\Gamma(1+(1 / 3))}{\Gamma((1 / 3)+(3 / 2))} \sim \pi(2 n+1) \hbar,
$$

where,

$$
\epsilon_{0}=E_{n}(\hbar, 0,1) \rightarrow\left(\frac{\Gamma[(3 / 2)+(1 / 3)] \sqrt{\pi} A}{\sin (\pi / 3) \Gamma[1+(1 / 3)]}\right)^{6 / 5},
$$

as $n \rightarrow \infty, n \hbar \rightarrow A>0[6]$.

\section{The absence of non-perturbative eigenval- ues}

We prove here that all the eigenvalues of our problem are perturbative since all the non-perturbative eigenvalues are non-modal. 
Let $-\pi \leq \arg (\beta)=\theta \leq 0,|\beta|=b>0$, (the condition $0 \leq \arg (\beta)=\theta \leq \pi$ is equivalent), $\lambda^{-2}=\hbar=b$, and let us consider the spectral equivalence by scaling:

$$
H(1,1, \beta) \sim b^{-1} H(b, 1, \beta / b)=\hbar^{-1} H\left(\hbar, 1, \hbar^{-1} \beta\right)=\hbar^{-1} H\left(\hbar, 1, \beta^{\prime}\right),
$$

where $\beta^{\prime}=\beta / b=\exp (i \theta)$

$$
H(\hbar, \alpha, \beta)=\hbar^{2} p^{2}+\alpha x^{2}+i \sqrt{\beta} x^{3},
$$

is the semiclassical three parameter Hamiltonian. We have the identity of the eigenvalues:

$$
E_{n, 1}(\beta)=E_{n}(1,1, \beta)=b^{-1} E_{n}(b, 1, \beta / b)=\hbar^{-1} E_{n}\left(\hbar, 1, \hbar^{-1} \beta\right)=\hbar^{-1} E_{n}\left(\hbar, 1, \beta^{\prime}\right) .
$$

We want to prove the non existence of non perturbative eigenvalues, i.e. that all the eigenvalues are semiclassical and perturbative. Let us recall [13] the 5 Stokes angular sectors of the complex $x$ plane, for $\beta \neq 0$,

$$
S_{k}=S_{k}(\arg (\beta))=\left\{z \in \mathrm{C} ;\left|\arg (i z)+\frac{1}{10} \arg (\beta)-\frac{2 k \pi}{5}\right|<\frac{\pi}{5}\right\},
$$

$-2 \leq k \leq 2$.

We discuss in detail the case with real parameter $\beta=b>0$, and the case at the boundary $\beta=b \exp (-i \pi)$, the other cases follow easily. We consider, for instance, the case $\beta=b / i$.

\section{The case of positive parameter}

Now, we fix $\arg (\beta)=0$, so that the semiclassical operator is,

$$
H(\hbar, 1,1)=\hbar^{2} p^{2}+x^{2}+i x^{3}=\hbar^{2} p^{2}+V(x) .
$$

The operator is defined by the $L^{2}$ condition at $\infty$ on the two sectors $S_{ \pm 1}(0)$. From the numerical range and the Hiesenberg incertainty principle, we know that the eigenvalues have positive real part $\Re(E)>0$.

The semiclassical quantization is based on the definition of a pair of classical turning points as functions of the energy $z_{ \pm}=z_{ \pm}(E), V\left(z_{ \pm}\right)=E$, defined on the half plane $\Re(E)>0$.

The $z_{ \pm}$classical turning points, stay on the half plane of conservation of the nodes, $\Im(z)<2 / 3$,

$$
\Re\left(z_{ \pm}\right)= \pm \frac{\sqrt{\Re(E)+y^{3}}}{\sqrt{1-3 y}}, \quad-(\Re(E))^{1 / 3}<y=\Im\left(z_{ \pm}\right)<\frac{1}{3}<y_{+}=\frac{2}{3} .
$$


Let us recall that the nodes are on the lower half plane, but there are no zeros on the strip $0 \leq \Im(z) \leq y_{+}=(2 / 3)$. We define the action as an analytic function of the energy,

$$
J(E)=\frac{1}{i} \oint_{\gamma} \sqrt{p_{E}^{2}(z)} d z
$$

where $p_{E}^{2}(z)=E-V(z), \gamma=\partial \Gamma$, oriented in the positive sense, and where $\Gamma$ is a convex, regular region, containing the two turning points $z_{ \pm}(E)$. The function $\sqrt{p_{E}^{2}(z)}$ has a cut on a arc of a curve joining the two branch points $z_{ \pm}$, where $p_{E}^{2}(z)>0$.

We have $J(E)=0$ only at $E=0$, where $z_{+}(0)=z_{-}(0)=0, V^{\prime}(0)=0$, and $\arg (J(E)) \rightarrow 0$ for $E \rightarrow 0$ on the half plane $\Re(E)>0$.

Let us consider an eigenvalue, $E=E(\hbar)=E_{n}(\hbar, 1,1), n \in \mathrm{N}$, of the semiclassical Hamiltonian (25), and its eigenfunction $\psi_{E}(x)$ with $n$ nodes on the lower complex half plane.

Lemma 2 All the eigenvalues of this problem are semiclassical. For any fixed $n \in \mathrm{N}$, we have, $E_{n}(\hbar)=(2 n+1) \hbar+O\left(\hbar^{2}\right)$, as $\hbar \rightarrow 0^{+}$, so that the eigenvalues of the original problem are perturbative, $E_{n, 1}(b) \rightarrow(2 n+1)$ as $b \rightarrow 0^{+}$.

\section{The Bohr-Sommerfeld quantization rule.}

In this case, the eigenfunction is proportional to the subdominant solution in the sector $S_{1}(0)$, with the WKB decreasing behavior,

$$
\frac{\psi_{E}^{\prime}(x)}{\psi_{E}(x)}=u_{E, \hbar}^{1}(x)+O(\hbar), u_{E, \hbar}^{1}(x)=-\frac{1}{\hbar} \sqrt{-p_{E}^{2}(x)}+\frac{1}{4} \frac{V(x)^{\prime}}{p_{E}^{2}(x)}
$$

where $p_{E}^{2}(x)=E-V(x)$, for $x>>\left|z_{+}(E)\right|$. This condition, and the analyticity of the exact and the approximate solution (26), the Bohr-Sommerfeld quantization rule for small $\hbar$,

$n=\frac{1}{2 i \pi} \oint_{\gamma} \frac{\psi_{E}^{\prime}(z)}{\psi_{E}(z)} d z=\frac{J(E)}{2 \pi \hbar}-\frac{1}{2}+O(\hbar)=\frac{J_{c}(\Re(E))}{2 \pi \hbar}-\frac{1}{2}+O(|\operatorname{Im}(E)|, \hbar)$,

where we have introduced the action $J$ and the classical action $J_{c}$,

$J(E)=\frac{1}{i} \oint_{\gamma} \sqrt{-p_{E}^{2}(z)} d z=J_{c}(\Re(E))+O(|\operatorname{Im}(E)|), \quad J_{c}(E)=2 \int_{x_{-}}^{x_{+}} p_{E}(x) d x$

$\gamma=\partial \Gamma$, and where $\Gamma$ is a convex, regular region, containing the two turning points $z_{ \pm}(E)$, with $x_{ \pm}=z_{ \pm}(\Re(E))$, and all the nodes of the exact eigenfunction. We have used the WKB approximation of the eigenfunction (29) at the first order with the cut of $p_{E}(x)$ on the interval of classical motion. This condition is sufficient to guarantee the perturbative nature of the eigenvalues. 


\section{Remark 4}

The stationary point $z=0$ of the potential is associated also to antibound states. This dependends on the possibility of the choice of another couple of sectors of subdominant solution. If we chose the dominant solution in sector $S_{1}(0)(26)$,

$$
u_{E, \hbar}^{1}(x)=+\frac{1}{\hbar} \sqrt{-p_{E}^{2}(x)}+\frac{1}{4} \frac{V(x)^{\prime}}{p_{E}^{2}(x)},
$$

we get the opposite value of the action,

$$
J(E)=\frac{1}{i} \oint_{\gamma} \sqrt{-p_{E}^{2}(z)} d z=-J_{c}(\Re(E))+O(|\operatorname{Im}(E)|) .
$$

In order to be more precise, we change variable $z= \pm i y$, and we get the Hamiltonian

$$
-H(\hbar, 1, \exp ( \pm i \pi))
$$

so that the choice of the couple of sectors $\left(S_{2}, S_{0}\right),\left(S_{0}, S_{-2}\right)$ gives almost negative eigenvalues for small $\hbar$. Analogously, the change of variable $z=$ $(2 / 3) i-y \sqrt{ \pm i}$, gives the top resonances operator

$$
-\frac{4}{27} \mp i H(\hbar, 1, \pm i)
$$

and non-perturbative eigenvalues, clearly non modal, defined on the sectors $\left(S_{-1}, S_{2}\right)$, and $\left(S_{-2}, S_{1}\right)$ respectively.

\section{A case at the boundary.}

Now, we fix $\arg (\beta)=-\pi$, so that the semiclassical Hamiltonian is real,

$$
H(\hbar, 1, \exp (-i \pi))=\hbar^{2} p^{2}+x^{2}+x^{3}=\hbar^{2} p^{2}+V(x) .
$$

Let us notice that the potential has a local minimum at 0 and a local maximum at $x=x_{m}=-2 / 3$, where $V_{m}=V\left(x_{m}\right)=4 / 27$ and $V^{\prime \prime}\left(x_{m}\right)=-2$. The operator is defined by the $L^{2}$ condition at $\infty$ on the two sectors $S_{ \pm 1}(-\pi)$. In this case, we have a standard problem of an unstable analytic single well, a typical shape resonance problem. Actually our operator is not a usual resonance operator since the physical problem is not complete, but this point does not matter now. We know that the eigenvalues are complex with negative imaginary part $\Im\left(E_{n}\right)<0$.

Let $\Im(E)<0$, the pair of classical turning points $z_{ \pm}=z_{ \pm}(E)$, stay on the lower half plane, where the other turning point $z_{0}$ stays on the upper half plane,

$$
0>\Im\left(z_{ \pm}\right)>-\sqrt{-\Im(E)} ; \quad \Im\left(z_{0}\right) \geq \sqrt{-\Im(E)} .
$$


We define the action as an analytic function of the energy,

$$
J(E)=\frac{1}{i} \oint_{\gamma} \sqrt{p_{E}^{2}(z)} d z
$$

where $p_{E}^{2}(z)=E-V(z), \gamma=\partial \Gamma$, oriented in the positive sense, and where $\Gamma$ is a convex, regular region, containing the two turning points $z_{ \pm}(E)$. The function $\sqrt{p_{E}^{2}(z)}$ has a cut on a arc of a curve joining the two branch points $z_{ \pm}$, where $p_{E}^{2}(z)>0$.

We have $J(E)=0$ only at $E=0$, where $z_{+}(0)=z_{-}(0)=0, V^{\prime}(0)=0$, and $\arg (J(E)) \rightarrow 0$ as $E \rightarrow 0$ on the half plane $\Im(E)<0$.

For $\hbar>0$ small, we have a well defined semiclassical quantization problem. Let us consider an eigenvalue, $E=E(\hbar)=E_{n}(\hbar, 1, \exp (-i \pi)) n \in \mathrm{N}$, of the semiclassical Hamiltonian (28), and its eigenfunction $\psi_{E}(x)$ with $n$ nodes on the lower complex half plane.

Lemma 2' All the eigenvalues of this problem are semiclassical. For any fixed $n \in \mathrm{N}$, we have, $E_{n}(\hbar)=(2 n+1) \hbar+O\left(\hbar^{2}\right)$, as $\hbar \rightarrow 0^{+}$, so that the eigenvalues of the original problem are perturbative, $E_{n, 1}(b \exp (-i \pi)) \rightarrow(2 n+1)$ as $b \rightarrow 0^{+}$.

\section{The quantization rule.}

In this case, the eigenfunction is proportional to the subdominant solution in sector $S_{1}(-\pi)$, with the WKB decreasing behavior,

$$
\frac{\psi_{E}^{\prime}(x)}{\psi_{E}(x)}=u_{E, \hbar}^{1}(x)+O(\hbar), u_{E, \hbar}^{1}(x)=-\frac{1}{\hbar} \sqrt{-p_{E}^{2}(x)}+\frac{1}{4} \frac{V(x)^{\prime}}{p_{E}^{2}(x)}
$$

where $p_{E}^{2}(x)=E-V(x)$, for $x>>\left|z_{+}(E)\right|$. This condition, and the analyticity of the exact and the approximate solution (29), the Bohr-Sommerfeld quantization rule for small $\hbar$,

$n=\frac{1}{2 i \pi} \oint_{\gamma} \frac{\psi_{E}^{\prime}(z)}{\psi_{E}(z)} d z=\frac{J(E)}{2 \pi \hbar}-\frac{1}{2}+O(\hbar)=\frac{J_{c}(\Re(E))}{2 \pi \hbar}-\frac{1}{2}+O(|\operatorname{Im}(E)|, \hbar),(30$

where we have introduced the action $J$ and the classical action $J_{c}$,

$J(E)=\frac{1}{i} \oint_{\gamma} \sqrt{-p_{E}^{2}(z)} d z=J_{c}(\Re(E))+O(|\operatorname{Im}(E)|), \quad J_{c}(E)=2 \int_{x_{-}}^{x_{+}} p_{E}(x) d x$

$\gamma=\partial \Gamma$, and where $\Gamma$ is a convex, regular region, containing the two turning points $z_{ \pm}(E)$, with $x_{ \pm}=z_{ \pm}(\Re(E))$, and all the nodes of the exact eigenfunction. We have used the WKB approximation of the eigenfunction (29) at the first order with the cut of $p_{E}(x)$ on the interval of classical motion. This condition is sufficient to garantee the perturbative nature of the eigenvalues. We have: $J_{c}(E)>0$ for $(4 / 27) \geq E>0$, analytic and monotonically 
growing, with $J(0)=0, J(4 / 27)=32 / 15$. The imaginary part of the turning points is small, $\Im\left(z_{ \pm}\right)=\Im(E) / V^{\prime}\left(x_{ \pm}\right)$, for fixed $\Re(E),(4 / 27)>\Re(E)>0$, $|\Im(E)|$ small. Thus, at first order in $\hbar$, the resonances are positive and are given by the Bohr-Sommerfeld semiclassical quantization,

$$
J_{c}\left(E_{n}\right)=2 \int_{x_{-}}^{x_{+}} \sqrt{E_{n}-V(x)} d x=2 \pi \hbar(n+(1 / 2))+O\left(\hbar^{2}\right) .
$$

Higher orders of semiclassical appoximation can give semiclassical approximations of the eigenvalues at any order of $\hbar$.

The quantization is completely specified by the Gamow condition for $x<<$ $-\left|x_{0}(0)\right|=-1$ :

$$
\frac{\psi_{E}^{\prime}(x)}{\psi_{E}(x)}=-i \frac{1}{\hbar} p_{E}(x)+\frac{1}{4} \frac{V(x)^{\prime}}{p_{E}^{2}(x)}+O(\hbar),
$$

equivalent to the $L^{2}$ condition on the sector $S_{-1}(-\pi)$.

This last condition discriminates our eigenvalues from the ones with $L^{2}$ condition on $S_{-2}(-\pi)$ in place of $S_{-1}(-\pi)$. For our purposes, it is sufficient to prove that our $J(E) \neq 0$ for $E \neq 0$. Indeed, in this analytic potential case, $J(E)=0$ only when $V^{\prime}\left(z_{ \pm}\right)=0$, and $z_{+}(E)=z_{-}(E)$. The only stationary points of this potential are at $x=0, x=-(2 / 3)$. In the case $E=0$, we have $z_{ \pm}(E)=0$, and $J(E)=0$, but, for $\Re(E)=4 / 27$, we have only one turning point $z_{-}(E) \sim-(2 / 3)$, for $|\Im(E)|$ small, the other $z_{+}(E)$ has positive real part, and $|J(E)| \neq 0$.

Since the number of nodes $n \in \mathrm{N}$ is fixed for $\hbar \rightarrow 0^{+}$, it is clear that $E=E_{n}(\hbar)=O(\hbar)$ in the same limit, and the original eigenvalue is bounded for small $|\beta|$, and

$$
E_{n}(1,1, \beta)=\hbar^{-1} E_{n}(\hbar, 1, \exp (-i \pi)) \rightarrow E_{n}(1,1,0)=2 n+1,
$$

as $|\beta| \rightarrow 0^{+}$, i.e. it is a perturbative eigenvalue.

Others eigenvalues, classified as anti-resonances, top resonances or antibound states, are obtained by changing the Stokes sectors of $L^{2}$ conditions at infinity.

Let us recall [13] the 5 Stokes angular sectors of the complex $z$ plane,

$$
S_{k}(\arg (\beta))=\left\{z \in \mathrm{C}, z \neq 0 ;\left|\arg (i z)+\frac{\arg (\beta)}{10}-\frac{2 k \pi}{5}\right|<\frac{\pi}{5}\right\}
$$

$-2 \leq k \leq 2$, where, in our case $\arg (\beta)=-\pi$. For instance,

$$
S_{1}(-\pi)=\left\{z \in \mathrm{C}, z \neq 0 ;-\frac{\pi}{5}<\arg (z)<\frac{\pi}{5}\right\} .
$$




\section{Remark 5}

The same operator with anti-Gamow condition at $-\infty$, is defined by $L^{2}$ conditions on the sectors $\left(S_{-2}(-\pi), S_{1}(-\pi)\right)$, and the eigenvalues $\hat{E}_{n}$ (antiresonances) are related to the original ones: $\hat{E}_{n}(1,1, \exp (-i \pi))=\bar{E}_{n}(1,1, \exp (-i \pi))=$ $E_{n}(1,1, \exp (i \pi))$.

Redefining $x \rightarrow-i x$, we get the negative eigenvalues $E_{n}^{\prime}(\hbar, 1, \exp (-i \pi))=$ $-E_{n}(\hbar, 1,1)$, of the operator with $L^{2}$ conditions on the two sectors $\left(S_{0}(-\pi), S_{2}(-\pi)\right)$ (antibound states). This eigenvalues are non perturbative, but are also non modal.

We now define the top resonances.

It is clear that the top resonances are defined as eigenvalues of Hamiltonians with different boundary conditions, and have nothing to do with the eigenvalues of our Hamiltonian. Actually, redefining $x \rightarrow(-2 / 3)-\sqrt{i} x$, we get the resonances $\mathcal{E}_{n}=(4 / 27)-i E_{n}(\hbar, 1,-i)$ of the operator defined by the $L^{2}$ conditions on the two sectors $\left(S_{2}(-\pi), S_{-1}(-\pi)\right)$. Redefining $x \rightarrow(-2 / 3)+\sqrt{-i} x$, we get the resonances $\mathcal{E}_{n}^{\prime}=(4 / 27)+i E_{n}(\hbar, 1, i)$ of the operator defined by the $L^{2}$ conditions on the two sectors $\left(S_{-2}(-\pi), S_{0}(-\pi)\right)$. It is clear that the top resonances are semiclassically connected with a different pair of inversion points $\left(\left(z_{-}, z_{0}\right)\right.$ in place of $\left.\left(z_{-}, z_{+}\right)\right)$.

We now go back to the general case, $-\pi \leq \arg (\beta)=\theta \leq 0$. The results are similar, the operator is always defined by the $L^{2}$ conditions on the sectors $\left(S_{-1}(\theta), S_{1}(\theta)\right)$, and $J(E)=J_{\beta}(E)$, for $|E|$ is the analytic continuation of the same function at $\beta=b \exp (-i \pi)$.

\section{An intermediary case}

Let us consider the case $\beta=-2 i$, so that the potential reads $V(x)=$ $x^{2}+\sqrt{2 i} x^{3}$. In this case $\Re(\mathcal{E})=\Re(\sqrt{i} E)>0$. We are mostly interested on the confinement of the turning point $z_{ \pm}$on the half complex plane of conservation of the nodes. We get the confinement of the turning points,

$$
x \pm=\Re\left(z_{ \pm}\right)= \pm \frac{\sqrt{\Re \mathcal{E}+y^{2}-2 y^{3}}}{\sqrt{1-6 y}}, \forall y=\Im\left(z_{ \pm}\right)<\frac{1}{6}<y_{+}=\frac{2}{3} \cos (\pi / 4) .
$$

Such results can be extended using complex $\hbar,|\arg (\hbar)|<\pi / 4$. This means that all the eigenvalues of our problem are perturbative.

Theorem 1' All the eigenvalues of the semiclassical operator are semiclassical, that is, are given by a Bohr-Sommerfeld condition for $\hbar$ small. As a consequence, all the eigenvalues are perturbative and are given by the unique continuation of the eigenvalues of Theorem 1. 


\section{The power law behavior at infinity}

We prove here the algebraic behavior of the eigenvalues for large parameter. We use the scaling formula:

$$
\sqrt{\alpha} E_{n, 1}(\beta)=E_{n, \alpha}(1)
$$

for $n \in \mathrm{N}$, where $\alpha=\beta^{-2 / 5}$. Let us recall that Theorem 4 , in the special case of $\alpha_{0}=0$, implies continuity and boundedness of each eigenvalue $E_{n, \alpha}(1)$ in the limit $\alpha \rightarrow 0$.

The analyticity, of type A, of the family of operators $H_{\alpha}(1)$ (see [2] Theorem 2.10 ), with the control of the nodes, and the simplicity of the spectrum, imply the stability at $\alpha=0$ and the $\alpha$-analyticity in a neighborhood of the origin of each eigenvalue $E_{n, \alpha}(1)$.

Therefore, if $\alpha=\beta^{-2 / 5}, \sqrt{\alpha} E_{n, 1}(\beta)=E_{n, \alpha}(1) \rightarrow E_{n, 0}(1)$ for $\beta \rightarrow \infty$. Thus, for $|\beta|=b$ large, $E_{n, \alpha}(\beta)$ grows as $b^{1 / 5}$, and has an algebraic singularity there:

$$
E_{n, 1}(\beta)=\beta^{1 / 5} E_{n, \beta^{-2 / 5}}(1) \sim \beta^{1 / 5} E_{n, 0}(1) .
$$

Let us notice that we have $\arg \left(E_{n, 1}(b \exp ( \pm i \pi))\right) \rightarrow \pm \pi / 5$, and

$$
\pm b^{-1 / 5} \Im\left(E_{n, 1}(b \exp ( \pm i \pi))\right) \rightarrow E_{n, 0}(1) \sin (\pi / 5)>0,
$$

as $b \rightarrow \infty$.

\section{Global analyticity, symmetry, and Padé summability on the cut plane}

Let $E(\beta)=E_{n, 1}(\beta), n=0,1,2, \ldots$,

$$
f(\beta)=\frac{E(\beta)-E(0)}{\beta},
$$

$f(\beta)$ is bounded holomorphic on the completed cut complex plane $\bar{C}_{c}=$ $\{\beta \in \mathrm{C} ; \beta \neq 0,|\arg (\beta)=\theta| \leq \pi\}$, (see Theorems 1-2-3'-4). Moreover, we 
have the symmetry of the eigenvalues: $E_{n, 1}(\beta)=\bar{E}_{n, 1}(\bar{\beta})$, so that we have, $f(\beta)=\bar{f}(\bar{\beta})$. For the Cauchy theorem, we have,

$$
f(\beta)=\frac{1}{2 i \pi} \oint_{\gamma} \frac{f(z)}{z-\beta}=\frac{1}{2 i \pi} \oint_{\gamma} \frac{1}{1-(\beta / z)} \frac{f(z)}{z} d z=\int_{0}^{\infty} \frac{1}{(1+\beta x)} \rho(x) d x,
$$

where $\gamma$ is any curve turning around $\beta$ in the positive way, we have the dispersion relation of a Stieltjes function, where

$$
\begin{aligned}
& \rho(1 / b)=-b\left(f\left(-b+i 0^{+}\right)-f\left(-b-i 0^{+}\right)\right) / 2 i \pi= \\
& =-b \Im f\left(-b+i 0^{+}\right) / \pi=\Im E_{n}\left(-b+i 0^{+}\right) / \pi \geq 0,
\end{aligned}
$$

for Lemma 1. We have the asymptotism to the formal power series:

$$
f(\beta) \sim \Sigma(\beta)=\sum_{j=0}^{\infty} a_{j}(-\beta)^{j}
$$

for $|\beta|$ small, where the

$$
a_{j}=\left|c_{j+1}\right|=\int_{0}^{\infty} x^{j} \rho(x) d x,
$$

are the moments of the measure $\rho(x) d x$. Thus, the moment problem

$$
a_{j}=\left|c_{j+1}\right|=\int_{0}^{\infty} x^{j} d \mu(x)
$$

has the solution $d \mu(x)=\rho(x) d x$. Because of the bound on the perturbation coefficients $\left|c_{j}\right|<A C^{j} j$ ! (see Theorem 1 and references [2], [3]), the Carleman theorem condition (see [20] page 330) is satisfied,

$$
\sum_{n}\left(1 / a_{n}\right)^{1 / 2 n}=\infty
$$

and the unicity of the solution $d \mu(x)=\rho(x) d x$.

Let us recall the definition of the diagonal Padé approximants $R_{n}^{n}(\beta)$ of the formal power series $\Sigma(\beta)=\sum_{j=0}^{\infty} a_{j}(-\beta)^{j}$, with partial sums $\Sigma^{N}(\beta)=$ $\sum_{j=0}^{N-1} a_{j}(-\beta)^{j}, \beta \in \mathrm{C}$. The diagonal Padé approximants, $R_{n}^{n}(\beta), n \geq 0$, are the rational fractions,

$$
R_{n}^{n}(\beta)=\frac{P_{n}(\beta)}{Q_{n}(\beta)}
$$

where $P_{j}(\beta), Q_{j}(\beta)$, are polynomials of degree $j$, with the condition $Q_{j}(0)=$ 1 , defined by the asymptotic condition, $\left|R_{n}^{n}(\beta)-\Sigma^{2 n+1}(\beta)\right|=O\left(\beta^{2 n+1}\right)$, for $|\beta| \rightarrow 0$. 
As a general result, the Padé approximants $R_{n}^{n}(\beta)$ on Stieltjes asymptotic expansions, don't have poles or zeros on the complex cut plane, and there converge

$$
R_{n}^{n}(\beta) \rightarrow f_{\mu}(\beta)=\int_{0}^{\infty} \frac{1}{(1+\beta x)} d \mu(x),
$$

where $d \mu$ is a measure solution of the moment problem (34). In this case, necessarily we have $d \mu(x)=\rho(x) d x$ and $f_{\mu}(\beta)=f(\beta)$.

Thus, we have the result:

\section{Theorem 5.}

The function

$$
f(\beta)=\frac{E(\beta)-E(0)}{\beta},
$$

is a Stieltjes function,

$$
f(\beta)=\int_{0}^{\infty} \frac{1}{(1+\beta x)} \rho(x) d x
$$

for $\beta$ on the cut complex plane, where

$$
\rho(1 / b)=\Im\left(E_{n}\left(-b+i 0^{+}\right)\right) / \pi>0,
$$

and,

$$
\ln (\rho(x))=-C^{-1} x(1+O(\ln (x) / x)),
$$

where $C^{-1}=8 / 15=2 B(2,3 / 2)=2 \int_{0}^{1} x \sqrt{1-x} d x$, [16] for large positive $x$. The diagonal Padé approximants of the perturbation series, converge to $f$,

$$
R_{n}^{n}(\beta) \rightarrow f(\beta)
$$

as $n \rightarrow \infty$, uniformly for $\beta$ on compacts of the cut complex plane.

\section{Proof.}

The inequality (36) is proved by the $\mathcal{P} \mathcal{T}$ symmetry of the eigenfunctions and eigenvalues, $E_{n}\left(-b+i 0^{+}\right)-E_{n}\left(-b-i 0^{+}\right)=2 i \Im\left(E_{n}\left(-b+i 0^{+}\right)\right)$and by Lemma 2. We only have to discuss the asymptotic behavior of the discontinuity function.

For the semiclassical behavior of the discontinuity (37), we consider the semiclassical scaling, where $b>0$ plays the role of semiclassical parameter, with the anti-Gamow condition at $+\infty$ :

$$
H(b, 1, \exp (i \pi)) \sim b H(1,1, b \exp (i \pi)) .
$$


In the case of the semiclassical operator $H(b, 1, \exp (i \pi))$, we have a "double well problem", with the barrier width $C^{-1}=8 / 15=2 \int_{0}^{1} x \sqrt{1-x} d x$, and $\hbar=b$. This value of the barrier, implies the behavior of $\rho(x)$, as $x \rightarrow$ $\infty$, as given in (37), and the behavior of the perturbation coefficients $c_{j}=$ $(-1)^{j} a_{j-1}, a_{j}=\int_{0}^{\infty} x^{j} d \rho(x), a_{j} \sim D C^{j} j$ !, as $j \rightarrow \infty$, for some $D>0$, compatible with the behavior:

$$
c_{j}=(-1)^{j-1} 4 \sqrt{15} C^{j}(2 \pi)^{-3 / 2} \Gamma(j+1 / 2)(1+O(1 / j)),
$$

for large $j$, obtained numerically [8] in the case $n=0$.

\section{Remark 4.}

We have proved, in a new way, that the eigenvalue $E(\beta)=E_{n, 1}(\beta), n=$ $0,1,2, \ldots$, is real and positive for positive $\beta$,

$$
E(\beta)=E(0)+\beta f(\beta)=E(0)+\beta \int_{0}^{\infty} \frac{1}{(1+\beta x)} \rho(x) d x \geq E(0),
$$

and $E_{n, 1}(\beta) \sim \beta^{1 / 5} E_{n, 0}(1)$ for large positive $\beta$.

\section{Aknowledgments}

We thank professor Pierre Duclos for useful discussions on this subject.

\section{References}

[1] Loeffel J, Martin A, Simon B, and Wightman A, Phys. Lett. B 30,656 (1969).

[2] Caliceti E, Graffi S, and Maioli M, Commun. Math. Phys. 75, 51 (1980).

[3] Caliceti E, J. Phys. A 333753 (2000).

[4] Caliceti E, Grecchi, and Maioli, M Commun. Math. Phys. 104, 163 (1986).

[5] Shin, K C, On the reality of eigenvalues for a class of PT-Symmetric oscillators, Commun. Math. Phys. 104, 229 (3), 543-564 (2002).

[6] Bender C M and Boettcher S : Real Spectra in Non-Hermitian Hamiltonian Having PT Symmetry, Phys. Rev. Lett. 80, 5243 (1998). 
[7] Bender C M, Boettcher S and Savage V M: Conjecture on interlacing of zeros in complex Sturm-Liouville problems, J. Math. Phys. 41, 63816387 (1984)

[8] Bender C M and Weniger S : Numerical evidence that the perturbation expansion for a non Hermitian PT-symmetric Hamiltonian is Stieljes, J. Math. Phys. 42, 2167-2183 (2001).

[9] Bouslaev V, Grecchi V: Equivalence of unstable anharmonic oscillators and double wells, J. Phys. A Math. Gen. 26, 5541-5549 (1993).

[10] Jones H F and Mateo J: An Equivalent Hermitian Hamiltonian for the non-Hermitian $-x^{4}$ Potential, Phys. Rev. D73 (2006) 085002.

C. M. Bender, D. C. Brody, J.-H. Chen, H. F. Jones, K. A. Milton, and M. C. Ogilvie: Equivalence of a Complex PT-Symmetric Quartic Hamiltonian and a Hermitian Quartic Hamiltonian with an Anomaly, Phys. Rev. D74 (2006) 025016.

H. F. Jones, J. Mateo and R. J. Rivers: On the Path-Integral Derivation of the Anomaly for the Hermitian Equivalent of the Complex PTSymmetric Quartic Hamiltonian, Phys. Rev. D74 (2006) 125022.

[11] Dorey P, Dunning C and Tateo R: Supersymmetriy and the spontaneus breakdown of PT-symmetry. J. Phys. A Math. Gen. 34, L391-L400 (2001);

Dorey P, Dunning C and Tateo R: Spectral equivalences, Bethe ansath equations, and reality propreties of PT-symmetric quantum mechanics, J. Phys. A Math. Gen. 34 (2001) 5697-5704.

[12] Aguilar J and Combes J M :A class of analytic Perturbations for the One-body Schrödinger Hamiltonians, Commun. Math. Phys. 22, 269279 (1971).

[13] Sibuya Y: Global theory of a second order linear ordinary differential equation with a polynomial coefficient, Chap. 7, Math. Studies 18, North Holland, (1975).

[14] Simon B Ann. Phys. 58, 76 (1970)

[15] Reed M, Simon B: Methods of modern mathematical physics, Vol. II and Vol. IV Academic Press, New York 1975-1978.

[16] Harrel E M II, Simon B Duke Math. j B 47,47 (1980).

[17] Dieudonné J: Calcul infinitésimal, (1968), Hermann, Paris. 
[18] Kato Tosio: Perturbation theory for linear operators, (1966) Springer, New York .

[19] Olver F W J : Asymptotics and special functions(1974) Academic Press, New York.

[20] Wall H S : Continued Fractions(1948) Van Nostrand, New York. 